We will try to publish authors' responses in the same edition with readers' comments. Time constraints might prevent this in some cases. The problem is compounded in a bimonthly journal where continuity of comment and redress are difficult to achieve. When the redress appears 2 months after the comment, 4 months will have passed since the original article was published. Therefore, we would suggest to our readers that their correspondence about published papers be submitted as soon as possible after the article appears.

\section{Thrombolysis in Acute Ischemic Stroke}

To the Editor: I was pleased to see the addition of the new feature, STEPped Care: An Evidenced-based Approach to Drug Therapy. It is the natural next step in the pursuit of a rational, considered approach to the medical literature. With the explosion of POEMs in multiple journals, applying the same rigor to new pharmaceutical modalities seems appropriate and forward-looking.

Unfortunately, the first article in the series falls below the standards set by proponents of evidence-based medicine. In their article on thrombolysis in acute ischemic stroke, Luisi and Hume ${ }^{1}$ consider the results of two large studies of recombinant tissue plasminogen activator (rt-PA) and stroke. The ECASS study describes a higher mortality and no significant improvement in functional outcomes in patients given rt-PA compared with placebo. ${ }^{2}$ The NINDS study showed an increase in the rate of symptomatic intracranial hemorrhage but no increase in overall mortality. ${ }^{3}$ Functional status was significantly better in the treatment group compared with the placebo group.

Attempting to reconcile variant findings into a cohesive recommendation is very difficult. It requires that differing results be accounted for and that care be taken not to favor the findings of one study over another without clear justification. Luisi and Hume did an excellent job of discussing the results of the studies. They fell short in their final interpretation, concluding that the use of thrombolytics in acute stroke is both safe and effective when used in a specified fashion.

The conclusion is surprising for a number of reasons. First, it does not reconcile the results of the component studies. It simply excludes the ECASS study (ie, 50 percent of the studies under consideration). The justification for exclusion is weak. Admittedly, several patients were excluded for protocol violations in the ECASS study. Interestingly, 60.6 percent of those violations resulted from reinterpretation of computed tomographic (CT) head scans. A tenet of evidence-based medicine is the applicability of the findings to the population at large. The radiologists involved in the study were specially trained in the evaluation of acute stroke. Given their variable interpretations despite the high level of training, how consistent would be the results of
CT scans performed in community hospitals? Radiology support in this setting is often patchy and, at best, involves a teleradiology link to a radiologist at another site. If one is to consider how these studies would play out in a real-life setting, the exclusion of the ECASS study seems unjustifiable.

Second, even if the ECASS can be excluded, making the recommendation for the use of rt-PA comes from one study only. Instead of appropriate caution for the introduction of a potentially lethal modality, the authors simply echo support for the published recommendations. ${ }^{4,5}$ Giving this support, without confirmation or reproduction of the findings, seems hard to justify.

Paul Hicks, MD Tucson, Ariz

\section{References}

1. Luisi A, Hume AL. Thrombolysis in acute ischemic stroke.J Am Board Fam Pract 1998;11:145-51.

2. Hacke $W$, Kaste $M$, Fieshi C, Toni D, Lesaffre E, von Kummer $\mathrm{R}$, et al. Intravenous thrombolysis with recombinant tissue plasminogen activator for acute hemispheric stroke. The European Cooperative Acute Stroke Study (ECASS). JAMA 1995;274:1017-25.

3. Tissue plasminogen activator for acute ischemic stroke. The National Institute of Neurological Disorders and Stroke rtPA Stroke Study Group. N Engl J Med 1995;333:1581-7.

4. Adams HP Jr, Brott TG, Furlan AJ, Gomez CR, Grotta J, Helgason CM, et al. Guidelines for thrombolytic therapy for acute stroke: a supplement to the guidelines for the management of patients with acute ischemic stroke. A statement for healthcare professionals from a Special Writing Group of the Stroke Council, American Heart Association. Stroke 1996;27:1711-8.

5. Practice advisory: thrombolytic therapy for acute ischemic stroke - summary statement. Report of the Quality Standards Subcommittee of the American Academy of Neurology. Neurology 1996;47:835-9.

The above letter was referred to the authors of the article in question, who offer the following reply.

To the Editor: We strongly agree with Dr. Hicks that further research into the use of rt-PA in acute ischemic stroke is urgently needed. Many questions have yet to be answered. While the appropriate use of thrombolytic agents in acute myocardial infarction has been well defined through many studies, information on recombinant tissue plasminogen activator (rt-PA) in acute ischemic stroke is based on two studies, only one of which was truly positive. Studies with streptokinase have been consistently negative. Unlike other topics and disease states to be discussed in this feature, few other therapeutic options can be offered to patients with an acute ischemic stroke. The critical question remains whether the recommendation to use throm- 